\title{
SCNS.01. Adenosine receptors and brain diseases: neuroprotection and neurodegeneration
}

\author{
CUNHA, R.A. ${ }^{1}$
}

${ }^{1}$ Center for Neuroscience and Cell Biology, Faculty of Medicine, University of Coimbra, Portugal.

Introduction: Caffeine is the most widely consumed psychoactive drug worldwide, but its potential beneficial effect for maintenance of proper brain functioning in adults has only recently begun to be adequately appreciated. Objectives: This has mainly resulted from the convergence of conclusions from epidemiological studies and from fundamental research in animal models: thus, epidemiological studies revealed an inverse association between the chronic consumption of caffeine and cognitive decline associated with aging or with Alzheimer's disease. Methods: This was paralleled by animal studies showing that caffeine prevented memory deficits as well as neurodegeneration. This ability of caffeine to normalize brain function has been extended to motor diseases and to neuropsychiatric conditions such as depression and attention deficits and hyperactivity disorders. Animal studies allowed indentifying the likely molecular target for caffeine, i.e. adenosine $A_{2 A}$ receptors $\left(A_{2 A} R\right)$. In fact, either the pharmacological or the genetic blockade of $A_{2 A} R$ mimics the neuroprotection, the mood and the memory normalisation afforded by chronic caffeine consumption. This involves a neuronal $A_{2 A} R$-mediated control of synaptic plasticity phenomena, and an astrocytic $A_{2 A} R$-mediated action. Conclusions: Notably, a novel optogenetic tool revealed that $A_{2 A} R$ activation seems sufficient to trigger memory impairment; this provides a key argument to foster translational efforts to exploit the prophylactic and therapeutic benefits of $A_{2 A} R$ antagonists as a novel strategy to manage neurodegenerative and neuropsychiatric disorders.

Funding: FCT, DARPA, MJFF, CAPES, Sanofi, Fundação Oriente

CUNHA, R.A. 2013. Adenosine receptors and brain diseases: neuroprotection and neurodegeneration, p.6. In: Oriá, Reinaldo Barreto; Andrade, Geanne Matos de; Bruin, Veralice Meireles S. de. I International Symposium in Neuroscience Meeting [Blucher Neuroscience Proceedings n.1 v.1]. São Paulo: Blucher, 2014 http://dx.doi.org/10.5151/isnm-sine2 